\title{
Geraniol, um componente dos óleos essenciais de plantas - um mapeamento científico de suas propriedades farmacológicas
}

Geraniol, a component of essential plant oils - a scientific mapping of its pharmacological properties

Geraniol, un componente de los aceites vegetales esenciales: un mapeo científico de sus propiedades farmacológicas

Recebido: 19/09/2021 | Revisado: 26/09/2021 | Aceito: 27/09/2021 | Publicado: 28/09/2021

Rodrigo Elísio de Sá

ORCID: https://orcid.org/0000-0002-1492-3995

Universidade Federal do Delta do Parnaíba, Brasil

E-mail: rodrigosa238@gmail.com

Lucicleia Dias Monteiro

ORCID: https://orcid.org/0000-0002-6904-4787

Universidade Federal do Delta do Parnaíba, Brasil E-mail: lucicleiabiomed@gmail.com

Priscila Costa Silva

ORCID: https://orcid.org/0000-0003-3605-2139

Universidade Federal do Delta do Parnaíba, Brasil E-mail: prycosta0309@gmail.com

Esley da Silva Santos

ORCID: https://orcid.org/0000-0003-4272-0543 Universidade Federal do Delta do Parnaíba, Brasil E-mail: esleeysantos@hotmail.com

Valentina Rhémily de Melo Vasconcelos

ORCID: https://orcid.org/0000-0002-3735-9936

Universidade Federal do Delta do Parnaíba, Brasil E-mail: valentinarhemily23@ hotmail.com

Rafaela de Brito Vieira

ORCID: https://orcid.org/0000-0001-5918-2804 Universidade Federal do Piauí, Brasil

E-mail: rafinhabritto18@gmail.com

Jhoana D'arc Lopes de Sousa

ORCID: https://orcid.org/0000-0001-5943-4012 Universidade Federal do Delta do Parnaíba, Brasil

E-mail: jhojhod1901@gmail.com

Antonia Luzia Lima do Nascimento

ORCID: https://orcid.org/0000-0001-9740-5669

Universidade Federal do Rio Grande do Sul, Brasil E-mail: antonialuzialima@gmail.com

Bruna Letícia Lima Carvalho

ORCID: https://orcid.org/0000-0003-2121-8239

Universidade Federal do Delta do Parnaíba, Brasil E-mail: brunaleticialc3@hotmail.com

Lucas Florencia da Silva

ORCID: https://orcid.org/0000-0002-7575-3643

Universidade Federal do Delta do Parnaíba, Brasil E-mail: lucasflorenci@gmail.com

\begin{abstract}
Resumo
A natureza oferece uma ampla diversidade química. Desse modo, as plantas representam uma importante fonte de novas substâncias devido, principalmente, aos metabólitos secundários. O Geraniol é um monoterpeno isoprenóide acíclico isolado de óleos essenciais de plantas aromáticas. As diversas atividades do geraniol sugerem que esta molécula poderia tratar várias doenças como um candidato a medicamento promissor. Para avaliar o potencial de geraniol aplicado em um ensaio clínico, esta revisão visa fornecer um resumo abrangente dos efeitos farmacológicos do geraniol. As publicações recuperadas Pubmed / NCBI, Science Direct, SCIELO e Google Scholar foram coletadas e resumidas entre os anos de 2013 a 2021. Em seguida, a aplicação potencial do geraniol como medicamento é
\end{abstract}


discutida com base em suas propriedades farmacológicas, incluindo antitumoral, antiinflamatória, atividades antioxidante e antimicrobiana e efeitos hepatoprotetores. Portanto, ressalta-se que a realização de futuras investigações pode contribuir significativamente para o acompanhamento de trabalhos com o GOH, possibilitando avaliar seu potencial farmacológico.

Palavras-chave: Geraniol; Óleo essencial; Farmacologia; Monoterpeno.

\begin{abstract}
Nature offers a wide chemical diversity. Thus, plants represent an important source of new substances, mainly due to secondary metabolites. Geraniol is an acyclic isoprenoid monoterpene isolated from aromatic plant essential oils. Geraniol's diverse activities suggest that this molecule could treat various diseases as a promising drug candidate. To assess the potential of geraniol applied in a clinical trial, this review aims to provide a comprehensive summary of the pharmacological effects of geraniol. The retrieved publications Pubmed / NCBI, Science Direct, SCIELO and Google Scholar were collected and summarized between the years 2013 to 2021 . Next, the potential application of geraniol as a medicine is discussed based on its pharmacological properties, including antitumor, anti-inflammatory, antioxidant and antimicrobial activities and hepatoprotective effects. Therefore, it is noteworthy that future investigations can significantly contribute to the follow-up of works with the $\mathrm{GOH}$, making it possible to assess its pharmacological potential.
\end{abstract}

Keywords: Geraniol; Essential oil; Pharmacology; Monoterpene.

\title{
Resumen
}

La naturaleza ofrece una amplia diversidad química. Por lo tanto, las plantas representan una fuente importante de nuevas sustancias, principalmente debido a los metabolitos secundarios. El geraniol es un monoterpeno isoprenoide acíclico aislado de aceites esenciales de plantas aromáticas. Las diversas actividades de Geraniol sugieren que esta molécula podría tratar diversas enfermedades como un candidato farmacológico prometedor. Para evaluar el potencial del geraniol aplicado en un ensayo clínico, esta revisión tiene como objetivo proporcionar un resumen completo de los efectos farmacológicos del geraniol. Las publicaciones recuperadas Pubmed / NCBI, Science Direct, SCIELO y Google Scholar se recopilaron y resumieron entre los años 2013 a 2021. A continuación, se discute la posible aplicación del geraniol como medicamento en función de sus propiedades farmacológicas, incluidas las antitumorales, antiinflamatorias, actividades antioxidantes y antimicrobianas y efectos hepatoprotectores. Por tanto, es de destacar que futuras investigaciones pueden contribuir significativamente al seguimiento del trabajo con el GOH, permitiendo evaluar su potencial farmacológico.

Palabras clave: Geraniol; Aceite esencial; Farmacología; Monoterpeno.

\section{Introdução}

As plantas medicinais são utilizadas na área da saúde desde tempos imemoriais. Desde a antiguidade, os produtos naturais desempenham importante papel em todo o mundo no tratamento e prevenção de doenças. Assim, diversos estudos buscam avaliar as propriedades terapêuticas destes metabólitos (Sofowora; Ogunbodede \& Onayade, 2013). De acordo com a Organização Mundial da Saúde (OMS), em 1985, 65\% da população mundial utilizavam as plantas medicinais como alternativa para os cuidados primários à saúde (Newman; Cragg, 2016).

A natureza oferece uma ampla diversidade química. Desse modo, as plantas representam uma importante fonte de novas substâncias devido, principalmente, aos metabólitos secundários. Tais metabólitos são utilizados para modular seu próprio organismo e para se defender do ataque de microrganismos e herbívoros. Estes metabólitos possuem uma constituição química altamente complexa, a qual apresenta um grande potencial terapêutico para doenças humanas (Naz et al., 2017).

Até meados do século XIX, os recursos terapêuticos eram compostos basicamente por plantas e extratos vegetais. Estes representavam a constituição da maioria dos medicamentos (Naveed et al., 2013). Graças a isso, no século XX, a indústria farmacêutica investiu mais em pesquisas com produtos naturais de origem vegetal (Newman; Cragg, 2016). Nos últimos anos, os organismos vegetais ganharam enorme destaque como fontes produtivas de substâncias com propriedades antitumorais e diversas outras atividades biológicas. Com isso, reativaram os interesses sociais e econômicos em explorar esse recurso. Hoje, os produtos naturais representam uma importante e crescente fonte de base para os produtos terapêuticos (Krishnan \& Padian, 2017).

Os óleos essenciais são substâncias voláteis, naturais, lipofílicas e complexos, caracterizados por um forte odor e são 
originados por plantas aromáticas como metabólitos secundários (Wadhwa et al.,2017). O óleo essencial é um dos produtos vegetais mais valiosos utilizados nas estratégias de medicamentos e tratamentos complementares. Farmacologicamente, são conhecidos por seu potencial antibacteriano, antiviral, antifúngico, analgésico, sedativo, anti-inflamatório, espasmolítico, dentre outros (Elshafie; Camele, 2017).

Esses óleos essenciais são constituídos principalmente de terpenos e fenilpropenos, mas a maioria dos óleos essenciais contém principalmente (90\%) terpenos (Olajuyigbe; Ashafa, 2014). Esses terpenos estão em diversas formas como mono, di, tri, tetraterpenos; em que monoterpenos têm dez carbonos, sesquiterpenos com quinze carbonos e diterpenos com vinte carbonos (Noriega, 2020). Esses terpenos são isolados de muitas plantas tradicionais e medicinais e estão sendo usados como a nova classe de moléculas farmacológicas bioativas para inúmeras condições patológicas (Stephane; Juleshttps, 2020).

Com isso, os produtos naturais têm se apresentado como uma excelente fonte de compostos com propriedades farmacológicas. Neste contexto, destacam-se os monoterpenos, especificamente os isoprenóides C10, que são compostos dietéticos encontrados nos óleos essenciais de muitas frutas, vegetais e ervas (Mendez-Perez et al., 2017). Os monoterpenos têm sido amplamente utilizados como aromatizantes e fragrâncias nas indústrias de alimentos e cosméticos, e como ingredientes ativos em muitos produtos farmacêuticos, devido às suas propriedades analgésicas, anti-inflamatórias e cicatrizantes (Koziol et al., 2014).

Geraniol (GOH) é um álcool monoterpênico acíclico derivado de óleos essenciais de plantas aromáticas (Lapczynski et al., 2008). Além de ser utilizado em diversos produtos comerciais, incluindo cosméticos e fragrâncias finas, o geraniol exerce um amplo espectro de atividades farmacológicas, como atividades antimicrobiana, anti-inflamatória, antioxidante, antiulcerativa e neuroprotetora (Rekha et al., 2013; Solórzano-Santos; Miranda-Novales, 2012).

Portanto, são necessários estudos para evidenciar informações na literatura sobre as atividades farmacológicas de metabólitos secundários de plantas, a exemplo do geraniol. Logo, este mapeamento tem como objetivo investigar as principais atividades farmacológicas descritas na literatura para a molécula.

\section{Metodologia}

No presente trabalho, foi elaborada uma revisão integrativa, este tipo de revisão representa uma importante contribuição acadêmica, pois sintetiza os resultados alcançados em diversos estudos de forma sistemática, ordenada e abrangente (Ercole, Melo \& Alcoforado, 2014). Além disso, revisões integrativas têm sido realizadas para avaliar as contribuições científicas de outros estudos, para que seja possível realizar uma análise crítica das publicações selecionadas (Bezerra, Do Espírito Santo, Monteiro \& Muto, 2020; Sousa, Prete, Gomes, De Castro \& Ribeiro, 2021). Os mapeamentos científicos possibilitam a avaliação de diferentes e atuais publicações na literatura científica, possibilitando realizar um estudo crítico e aprofundado acerca dos trabalhos disponíveis e condizentes com o tema proposto (Rother, 2007; Prodanov \& Freitas, 2013).

Atendendo ao objetivo do trabalho, os seguintes descritores foram empregados na busca das publicações: geraniol sozinho ou combinado com antioxidante, antiinflamatório, antidiabético, antifúngico, antitumoral e hepatoprotetor, como palavras-chave para as pesquisas de literatura. Os bancos de dados usados para a pesquisa incluíram Pubmed / NCBI, Science Direct, SCIELO e Google Scholar. Nossos critérios de inclusão consistiram em artigos experimentais publicados em periódicos internacionais, com texto completo disponível, publicados entre janeiro de 2013 a julho de 2021. Esses critérios visavam restringir nossas descobertas a publicações relacionadas ao tema de interesse. Resumos e publicações sem a avaliação das atividades biológicas foram excluídos do estudo. A análise e síntese dos dados são apresentadas de forma sucinta e descritiva, o que permite a sua observação, contagem e descrição, visando reunir um conjunto de informações sobre o assunto abordado. 


\section{Resultados e Discussão}

\subsection{Atividade antifúngica}

Candida albicans é um fungo que acomete principalmente pacientes imunocomprometidos e é responsável por uma alta taxa de mortalidade (Rekha; Selvakumar, 2014). Um recente estudo mostrou que o tratamento com GOH enfraqueceu o potencial de espécies Candida albicans. Administração oral de GOH mostrou um efeito inibitório na formação de biofilme de fungos patogênicos e na morfogênese das hifas. GOH destruiu a função da parede celular regulando negativamente a atividade da ATPase da membrana plasmática e reduzindo os níveis de ergosterol. Além disso, o GOH destruiu a função mitocondrial, quebrou a homeostase do ferro e mitigou a toxicidade (Singh; Fatima; Hameed, 2016). Outro estudo mostrou que o mínimo inibitório a concentração de GOH é de $16 \mu \mathrm{g} / \mathrm{mL}$ contra C. albicans e o efeito antimicótico de GOH é dependente da concentração. O teste de interferência morfológica demonstra que este composto possui efeito supressor na formação de pseudo-hifas e clamidoconídios (Leite et al., 2015).

Trichophyton rubrum é um dos fungos mais comuns encontrados em casos de dermatofitose crônica (Da Silva et al., 2014). Uma alta concentração de GOH pode suprimir o crescimento micelial e fúngico e também inibir germinação de conídios. Após tratamento com GOH, a infecção por T. rubrum sofreu inibição da biossíntese de ergosterol para destruir a parede celular e a membrana celular (Pereira et al., 2015).

\subsection{Efeitos hepatoprotetores}

GOH pode aliviar a esteatohepatite não alcoólica em ratos induzido por dieta com deficiência de metioninecolina por meio de $200 \mathrm{mg} / \mathrm{kg}$ desse composto como suplemento oral. Neste modelo, a administração de GOH mostrou um efeito inibidor nas pontuações histológicas, fibrose e apoptose no fígado. A nível molecular, o GOH parece inibir as atividades da alanina aminotransferase (ALT) e aspartato aminotransferase (AST) no soro. Somado a isso, destaca-se que este protege a função mitocondrial hepática através da supressão de ROS mitocondrial hepática, atividade da enzima da cadeia de transporte de elétrons mitocondrial e conteúdo de DNA mitocondrial. Ademais, a administração de GOH atenua a formação de malondialdeído e 3-nitrotirosina e inibe a expressão de iNOS e citocromo P450 2E1 no fígado. Além disso, o GOH mostra um efeito inibitório sobre a inflamação no fígado com a inibição do TNF- $\alpha$ e expressão de IL-6 e atividade de mieloperoxidase (Chen et al., 2017).

Outrossim, o GOH suprimiu notavelmente o nível de ALT no soro, que regula a recuperação da função das células do fígado (Canbek et al., 2017). Este é um composto natural que estimula notavelmente a regeneração do fígado, conforme mostrado em um estudo com um modelo de camundongo, onde foi injetado $100 \mathrm{mg} / \mathrm{kg}$ desta molécula. Em relação ao mecanismo, GOH aumenta a expressão de mRNA de NF-אB, TNF- $\alpha$ e IL-6 e níveis de proteína, e inibe os níveis de proteína HSP27 e HSP60 (Ceyhan; Canbek, 2017).

\subsection{Efeitos Antidiabéticos}

O diabetes é um distúrbio metabólico que se apresenta com alto nível de açúcar no sangue e pode potencialmente levar a várias complicações. Em um modelo de rato diabético induzido por estreptzotocina, a administração de GOH por 45 dias diminuiu a glicose plasmática e a hemoglobina HbA1C e pôde restaurar a resposta à insulina. A atividade de muitas enzimas envolvidas na produção e utilização de glicose foram restaurados, levando a uma melhoria no metabolismo de carboidratos e à restauração da homeostase da glicose (Babukumar et al., 2017). Outro estudo em um modelo de rato diabético induzido por STZ mostrou que o tratamento com GOH tem um efeito protetor sobre a neuropatia diabética e melhorou as funções sensoriais e motoras, diminuindo atividades enzimáticas (ou seja, complexos I-III, succinato desidrogenase, e citrato sintase) no citosol do nervo ciático e em regiões selecionadas do cérebro. Somado a isso, o GOH pode aliviar os níveis de 
cálcio citosólico e atividade da acetilcolinesterase (Prasad, 2014).

\subsection{Atividades anti-inflamatória e antioxidante}

A inibição da resposta inflamatória e do estresse oxidativo evita danos aos órgãos, especialmente no cérebro. A peroxidação lipídica e a reação inflamatória causada pelo 12-O-tetradecanoilforbol 13-acetato (TPA) pode ser significativamente diminuída pelo $\mathrm{GOH}$. Investigações posteriores demonstraram que o $\mathrm{GOH}$ promove o metabolismo de células inflamatórias, aumenta o conteúdo de GSH e estimula as atividades de enzimas antioxidantes. Além disso, GOH exerce

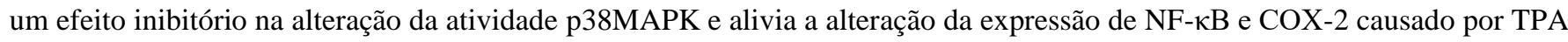
(Khan et al., 2013). Outro estudo também afirmou que o GOH poderia inibir a inflamação por meio da inibição da COX-2 (SU et al., 2010). GOH tem um efeito inibitório na resposta inflamatória induzida por pamidronato, estimulando a produção de IL10. Ademais, o GOH não mostrou efeito tóxico sobre os monócitos e não impactou a produção de TNF- $\alpha$ (Murbach Teles Andrade et al., 2014).

Um estudo testou parâmetros bioquímicos e oxidativos hepáticos após o tratamento GOH em ratos Wistar e concluiu que $\mathrm{GOH}$ pode funcionar como um antioxidante durante a aromaterapia. A administração de GOH foi negativamente correlacionada com o nível de colesterol total, mas não houve diferença na glicemia, proteína triacilglicerol e níveis de ureia (Jayachandran; Chandrasekaran; Namasivayam, 2015).

\subsection{Atividades citotóxicas e antitumorais}

O câncer de cólon ocupa o quarto lugar na causa de mortalidade por câncer em todo o mundo (Zhang et al.,2018). Um estudo recente demonstrou que GOH pode inibir significativamente o crescimento na linhagem celular de câncer de cólon-205 com um valor de CI50 de $20 \mu \mathrm{M}$. A Coloração DAPI indicou que GOH induz apoptose, e um ensaio de Western blot confirmou que o $\mathrm{GOH}$ regula positivamente o Bax e diminui o Bcl-2. Bax é um regulador de pró-apoptose e Bcl-2 é um regulador de anti-apoptose. Além disso, a GOH induz danos ao DNA e parada do ciclo celular em células de cólon-205 (Qi et al., 2018). O carcinoma endometrial é um câncer comum entre as mulheres em todo o mundo. Vale a pena pesquisar novos candidatos a medicamentos para o tratamento do carcinoma endometrial.

Uma pesquisa recente mostrou que o tratamento com GOH pode suprimir o carcinoma endometrial ao inibir oncogenes e ativar genes supressores de tumor em um modelo de rato Wistar fêmea induzido por N-metil-N2-nitro-Nnitrosoguanidina a $150 \mathrm{mg} / \mathrm{kg}$. O efeito de supressão do GOH foi mais eficaz no grupo pré-tratado. Os resultados de PCR em tempo real mostram que GOH inibe a transcrição de K-ras, proteína quinase ativada por mitogênio (MAPK), PI3K e $\beta$ catenina, e os resultados de Western blot mostram que o GOH aumenta a fosfatase e fosfatase e tensina homóloga deletada no cromossomo dez (PTEN), receptores de progesterona e expressão da proteína E-caderina. O efeito na angiogênese foi verificado in vivo: a administração de GOH a camundongos BALB / c reduz o número de Ki67-positivos em células e através da atenuação da expressão de fator de crescimento endotelial vascular (VEGFR2) (Wittig et al.,2015).

\subsection{Outros efeitos}

GOH mostra um efeito antiarrítmico em arritmias induzidas por ouabaína em modelos de mamíferos. Neste mesmo estudo, observou-se que esta molécula alivia arritmias induzidas por ouabaína através da regulação negativa da força contrátil do coração. A administração de GOH bloqueia a corrente transitória de potássio $(\mathrm{K}+)(59,7 \%)$, a corrente $\mathrm{K}+$ de não inativação $(39,2 \%)$, e a corrente do retificador de entrada $\mathrm{K}+(33,7 \%)$, levando a uma ação potencial, que aumentou em $50 \%$. Constatou-se também diminuição da pressão ventricular esquerda (83\%) e frequência cardíaca $(16,5 \%)$. Somado a isso, também foi demonstrado que GOH prolongou o início de arritmias induzidas por ouabaína em $128 \%$ e diminuiu a tensão de 
repouso em 30\% (De Menezes-Filho et al., 2014).

Este composto mostra excelente atividade anti-ulcerogênica no estômago e na mucosa duodenal. Após a administração oral em ratos, uma dose de 7,50 mg / kg de GOH foi capaz de induzir um aumento do conteúdo de glutationa (GSH) e uma diminuição dos níveis de mieloperoxidase. Além disso, a molécula reduz a lesão ulcerativa duodenal induzida por isquemia / reperfusão em $71 \%$ e as úlceras duodenais induzidas por cisteamina em $68 \%$. Adicionalmente, protege o estômago e mucosa duodenal, aumentando as atividades de proteção fatores, incluindo prostaglandinas endógenas e óxido nítrico. O mecanismo de proteção também envolve o aumento de secreção de muco com propriedades antioxidantes (De Carvalho et al., 2014).

GOH tem um efeito protetor na lesão de colite induzida por ácido trinitro-benzeno-sulfônico (TNBS). Especificamente, induzido por TNBS em ratos com colite, e tratados com o composto por 11 dias mostraram uma melhora de sintomas clínicos (por exemplo, perda de peso, edema de cólon, ulceração e índices de massa do cólon / baço). GE e / ou sulfassalazina poderia prevenir o aumento dos níveis de óxido nítrico e peróxido de lipídio e restaurar a atividade antioxidante no modelo de rato com colite. A nível molecular, a molécula inibe apoptose e a infiltração de células inflamatórias por redução dos níveis de caspase-3, molécula de adesão intercelular-1 e mieloperoxidase (Soubh; Abdallah \& El-Abhar, 2015).

A administração oral de GOH por 3 semanas mostrou um efeito do tipo antidepressivo em estresse crônico imprevisível leve (CUMS) em modelos de ratos e encurtou significativamente o tempo imóvel em testes de suspensão da cauda. Mais especialmente, o composto diminuiu os níveis de IL-1 $\beta$ pró-inflamatórios induzidos por CUMS, resultando em a melhora do comportamento depressivo. Além disso, a molécula ajustou a ligação de nucleotídeos e a expressão de família de receptores semelhantes a domínios de oligomerização contendo 3 inflamassomas contendo domínio de pirina (Deng et al., 2015).

Cabe destacar que o composto ainda demonstra potencial frente a Trichomonas vaginalis, matando o parasita. Além disso, destruiu a membrana nuclear e os núcleos, levando ao acúmulo de cromatina e um grande número de vacúolos aparecendo no citoplasma. Tão logo, os ribossomos foram reduzidos, as organelas foram desintegradas e a membrana celular foi até danificada. Curiosamente, GOH não mostrou um efeito inibitório de uma maneira dependente do tempo (Dai et al., 2016).

\section{Considerações Finais}

Atualmente, compostos isolados de produtos naturais estão ganhando aceitação como potencialmente promissores complementares e alternativos medicamentos para o tratamento de várias doenças. Geralmente, tais compostos podem ter como alvo DNA, mRNA, proteína e até microRNA. O acúmulo de evidências nas últimas décadas indicou que o GOH é um composto botânico puro sem efeitos adversos, exercendo diversas propriedades, regulando principalmente a expressão de proteínas, sugerindo que esta biomolécula poderia se tornar uma nova droga candidata a tratar várias doenças. $\mathrm{O}$ efeito citotóxico desse composto nas células cancerosas indica que este pode tratar o câncer e reduzir a mortalidade de pacientes com essa condição patológica. Os efeitos antiinflamatórios e oxidativos de GOH indicam que este também pode proteger os danos aos órgãos e tratar a colite ulcerosa. A atividade antifúngica do GOH indica que este poderia proteger os pacientes de infecções fúngicas. O efeito antidiabético de GOH demonstra o potencial para tratar diabetes. Entretanto, os efeitos anticâncer do GOH devem ser esclarecidos in vivo em mais modelos animais e, posteriormente, em pacientes humanos, para confirmar o efeito inibitório do GOH na malignidade. Além disso, vale a pena explorar a base molecular subjacente à farmacologia, ações (por exemplo, atividades antimicrobianas e antiarrítmicas). A falta de informações sobre os alvos definitivos do GOH seria um motivo contra a aplicação em ensaios clínicos.

Portanto, ressalta-se que a realização de futuras investigações pode contribuir significativamente para o 
acompanhamento de trabalhos com o GOH, possibilitando avaliar seu potencial farmacológico, pois o mesmo apresenta diversas atividades biológicas promissoras.

\section{Referências}

Babukumar, S., Vinothkumar, V., Sankaranarayanan, C. \& Srinivasan, S. (2017). Geraniol, a natural monoterpene, ameliorates hyperglycemia by attenuating the key enzymes of carbohydrate metabolism in streptozotocin-induced diabetic rats. Pharmaceutical biology, 55(1), $1442-1449$.

Bezerra, D. R. C., do Espírito Santo, F. H., Monteiro, J. K. D. M. F., \& Muto, T. S. (2020). Os vulneráveis no período do COVID-19: uma revisão integrativa de literatura. Research, Society and Development, 9(10), e4699108860-e4699108860. https://rsdjournal.org/index.php/rsd/article/view/8860/7816.

Canbek, M., Uyanoglu, M., Canbek, S., Ceyhan, E., Ozen, A., Durmus, B. \& Turgak, O. (2017). The effect of geraniol on liver regeneration after hepatectomy in rats. in vivo, 31(2), 209-213.

Ceyhan, E. \& Canbek, M. (2017). Determining the Effects of Geraniol on Liver Regeneration Via the Nuclear Factor $\kappa$ B Pathway After Partial Hepatectomy. Alternative therapies in health and medicine, 23(3), 38-45.

Chen, J., Fan, X., Zhou, L. \& Gao, X. (2016). Treatment with geraniol ameliorates methionine-choline-deficient diet-induced non-alcoholic steatohepatitis in rats. Journal of gastroenterology and hepatology, 31(7), 1357-1365.

da Silva, B. C. M., Paula, C. R., Auler, M. E., Ruiz, L. D. S., Dos Santos, J. I., Yoshioka, M. C. N., Fabris, A., Castro, L. G. M., Duarte, A. J. S. \& Gambale, W. (2014). Dermatophytosis and immunovirological status of HIV-infected and AIDS patients from Sao Paulo city, Brazil. Mycoses, 57(6), $371-376$.

da Silva, R. E., Ribeiro, F. D. O. S., de Araújo, G. S., Iles, B., Pessoa, O. D. L., de Araújo, A. R. \& dos Santos Soares, M. J. (2021). Biological properties of Oncocalyxone A: a review. Research, Society and Development, 10(4), e57810414343-e57810414343.

Dong, G. \& Golden, S. S. (2008). How a cyanobacterium tells time. Current opinion in microbiology, 11(6), 541-546.

Elshafie, H. S. \& Camele, I. (2017). An overview of the biological effects of some mediterranean essential oils on human health. BioMed research international, 2017.

Ercole, F. F., Melo, L. S. D. \& Alcoforado, C. L. G. C. (2014). Revisão integrativa versus revisão sistemática. Revista Mineira de Enfermagem, 18(1), 9-12.

Jayachandran, M., Chandrasekaran, B. \& Namasivayam, N. (2015). Geraniol attenuates oxidative stress by Nrf2 activation in diet-induced experimental atherosclerosis. Journal of basic and clinical physiology and pharmacology, 26(4), 335-346.

Khan, A. Q., Khan, R., Qamar, W., Lateef, A., Rehman, M. U., Tahir, M., Ali, F., Hamiza, O. O., Hasan, S. K. \& Sultana, S. (2013). Geraniol attenuates 12-Otetradecanoylphorbol-13-acetate (TPA)-induced oxidative stress and inflammation in mouse skin: Possible role of p38 MAP Kinase and NF- $\mathrm{B}$. Experimental and molecular pathology, 94(3), 419-429.

Koziol, A., Stryjewska, A., Librowski, T., Salat, K., Gawel, M., Moniczewski, A. \& Lochynski, S. (2014). An overview of the pharmacological properties and potential applications of natural monoterpenes. Mini reviews in medicinal chemistry, 14(14), 1156-1168.

Leite, M. C. A., de Brito Bezerra, A. P., de Sousa, J. P. \& de Oliveira Lima, E. (2015). Investigating the antifungal activity and mechanism (s) of geraniol against Candida albicans strains. Medical mycology, 53(3), 275-284.

Mendez-Perez, D., Alonso-Gutierrez, J., Hu, Q., Molinas, M., Baidoo, E. E., Wang, G., Chan, L. J. G., Adams, P. D., Petzold, C. J., Keasling, J. D. \& Lee, T. S. (2017). Production of jet fuel precursor monoterpenoids from engineered Escherichia coli. Biotechnology and bioengineering, 114(8), 1703-1712.

Murbach Teles Andrade, B. F., Conti, B. J., Santiago, K. B., Fernandes, A. \& Sforcin, J. M. (2014). C ymbopogon martinii essential oil and geraniol at noncytotoxic concentrations exerted immunomodulatory/anti-inflammatory effects in human monocytes. Journal of Pharmacy and Pharmacology, 66(10), 1491-1496.

Naveed, R., Hussain, I., Tawab, A., Tariq, M., Rahman, M., Hameed, S., Mahmood, M.S., Siddique, A. B., Iqbal., M. Antimicrobial activity of the bioactive components of essential oils from Pakistani spices against Salmonella and other multi-drug resistant bacteria. BMC Complementary and Alternative Medicine, 13 (1) (2013) 265. https://link.springer.com/article/10.1186/1472-6882-13-265

Naz, R., Ayub, H., Nawaz, S., Islam, Z. U., Yasmin, T., Bano, A., \& Roberts, T. H. (2017). Antimicrobial activity, toxicity and anti-inflammatory potential of methanolic extracts of four ethnomedicinal plant species from Punjab, Pakistan. BMC complementary and alternative medicine, 17(1), 1-13. https://link.springer.com/article/10.1186/s12906-017-1815-z.

Newman, D. J. \& Cragg, G. M. (2016). Natural products as sources of new drugs from 1981 to 2014. Journal of natural products, 79(3), 629-661.

Noriega, P. (2020). Terpenes in Essential Oils: Bioactivity and Applications. In Terpenes and Terpenoids. IntechOpen.

Olajuyigbe, O. \& Ashafa, A. (2014). Chemical composition and antibacterial activity of essential oil of Cosmos bipinnatus Cav. leaves from South Africa. Iranian journal of pharmaceutical research: IJPR, 13(4), 1417.

Pereira, F. D. O., Mendes, J. M., Lima, I. O., Mota, K. S. D. L., Oliveira, W. A. D. \& Lima, E. D. O. (2015). Antifungal activity of geraniol and citronellol, two monoterpenes alcohols, against Trichophyton rubrum involves inhibition of ergosterol biosynthesis. Pharmaceutical biology, 53(2), 228-234.

Prasad, S. N. (2014). Protective effects of geraniol (a monoterpene) in a diabetic neuropathy rat model: attenuation of behavioral impairments and biochemical perturbations. Journal of neuroscience research, 92(9), 1205-1216. 
Research, Society and Development, v. 10, n. 12, e508101220805, 2021

(CC BY 4.0) | ISSN 2525-3409 | DOI: http://dx.doi.org/10.33448/rsd-v10i12.20805

Prodanov, C. C. \& De Freitas, E. C. (2013). Metodologia do trabalho científico: métodos e técnicas da pesquisa e do trabalho acadêmico-2a Edição. Editora Feevale.

Qi, F., Yan, Q., Zheng, Z., Liu, J., Chen, Y. \& Zhang, G. (2018). Geraniol and geranyl acetate induce potent anticancer effects in colon cancer Colo-205 cells by inducing apoptosis, DNA damage and cell cycle arrest. $J$ BUON, 23(2), 346-352.

Rekha, K. R. \& Selvakumar, G. P. (2014). Gene expression regulation of Bcl2, Bax and cytochrome-C by geraniol on chronic MPTP/probenecid induced C57BL/6 mice model of Parkinson's disease. Chemico-biological interactions, 217, 57-66.

Rekha, K. R., Selvakumar, G. P., Sethupathy, S., Santha, K. \& Sivakamasundari, R. I. (2013). Geraniol ameliorates the motor behavior and neurotrophic factors inadequacy in MPTP-induced mice model of Parkinson's disease. Journal of Molecular Neuroscience, 51(3), 851-862.

Singh, S., Fatima, Z. \& Hameed, S. (2016). Insights into the mode of action of anticandidal herbal monoterpenoid geraniol reveal disruption of multiple MDR mechanisms and virulence attributes in Candida albicans. Archives of microbiology, 198(5), 459-472.

Sofowora, A., Ogunbodede, E., \& Onayade, A. (2013). The role and place of medicinal plants in the strategies for disease prevention. African journal of traditional, complementary and alternative medicines, 10(5), 210-229. https://www.ajol.info/index.php/ajtcam/article/view/92333.

Soubh, A. A., Abdallah, D. M.; \& El-Abhar, H. S. (2015). Geraniol ameliorates TNBS-induced colitis: Involvement of Wnt/ $\beta$-catenin, p38MAPK, NFkB, and PPAR $\gamma$ signaling pathways. Life sciences, 136 (8), 142-150.

Sousa, C. J. P., Prete, A. C. L., Gomes, A. G. P., de Castro, E. F. R. \& Ribeiro, C. H. M. A. (2021). Adverse drug events in hospitalized patients in Brazil: Integrative literature review. Research, Society and Development, 10(4), e3410413818-e3410413818.

Stephane, F. F. Y. \& Juleshttps, B. K. J. (2020). Terpenoids as important bioactive constituents of essential oils. In Essential Oils-Bioactive Compounds, New Perspectives and Applications. IntechOpen.

Su, Y. W., Chao, S. H., Lee, M. H., Ou, T. Y. \& Tsai, Y. C. (2010). Inhibitory effects of citronellol and geraniol on nitric oxide and prostaglandin E2 production in macrophages. Planta medica, 76(15), 1666-1671.

Xue-Y., D., Jin-S. X., Hong-Y. L,Zhan-Q.M., \& Qiang., F. R. (2015). Geraniol produces antidepressant-like effects in a chronic unpredictable mild stress mice model. Physiology \& Behavior, 152 (9), 264-271.

Wadhwa, G., Kumar, S., Chhabra, L., Mahant, S. \& Rao, R. (2017). Essential oil-cyclodextrin complexes: An updated review. Journal of inclusion phenomena and macrocyclic chemistry, 89(1), 39-58.

Wittig, C., Scheuer, C., Parakenings, J., Menger, M. D. \& Laschke, M. W. (2015). Geraniol suppresses angiogenesis by downregulating vascular endothelial growth factor (VEGF)/VEGFR-2 signaling. PLoS One, 10(7), e0131946.

Zhang, Z., Zhao, S., Li, X., Zhuo, X., Zhang, W., Nie, Q., Wang, S., Yan, L. \& Sun, Y. (2018). Amentoflavone inhibits osteoclastogenesis and wear debrisinduced osteolysis via suppressing NF-KB and MAPKs signaling pathways. Planta medica, 84(11), 759-767. 